\title{
Holographic butterfly velocities in brane geometry and Einstein-Gauss-Bonnet gravity with matters
}

\author{
Wung-Hong Huang" \\ Department of Physics, National Cheng Kung University, No.1, University Road, Tainan 701, Taiwan
}

(Received 10 November 2017; published 23 March 2018)

\begin{abstract}
In the first part of the paper we generalize the butterfly velocity formula to anisotropic spacetime. We apply the formula to evaluate the butterfly velocities in M-branes, D-branes, and strings backgrounds. We show that the butterfly velocities in M2-branes, M5-branes and the intersection M2 $\perp$ M 5 equal to those in fundamental strings, D4-branes and the intersection F1 $\perp$ D4 backgrounds, respectively. These observations lead us to conjecture that the butterfly velocity is generally invariant under a doubledimensional reduction. In the second part of the paper, we study the butterfly velocity for Einstein-GaussBonnet gravity with arbitrary matter fields. A general formula is obtained. We use this formula to compute the butterfly velocities in different backgrounds and discuss the associated properties.
\end{abstract}

DOI: $10.1103 /$ PhysRevD.97.066020

\section{INTRODUCTION}

Quantum chaos is naturally characterized by the commutator $[W(t, x), V(0)]$ which measures the dependence of a later operator $W(t, x)$ on an earlier perturbation $V(0)$. The strength of the associated butterfly effect can be described by $[1,2,3]$

$$
\left\langle[W(t, x), V(0)]^{2}\right\rangle_{T} \sim e^{\lambda\left(t-t_{*}-\frac{|x|}{v_{B}}\right)}
$$

where $t_{*}$ is a time scale called the scrambling time at which the commutator grows to be $\mathcal{O}(1)$. The butterfly velocity $v_{B}$ characterizes the speed at which the perturbation grows. The Lyapunov exponent $\lambda$ measures the rate of growth of chaos, and it is bounded by temperature $\mathrm{T}: \lambda \leq 2 \pi \beta$, where $\beta=\frac{1}{T}$. The inequality saturates for the thermal systems that have a dual holographic black hole described by Einstein gravity [4].

Butterfly effects in quantum chaos have been extensively studied recently in the holographic theories [5-26]. In the holographic approach the butterfly velocity is identified by the velocity of the shock wave which describes how the perturbation spreads in space $[8,10,11]$. The method of finding the shock-wave velocity for the general spacetime with matters has been described many years ago in [27] and was used to obtain the butterfly velocity. In this paper we

\footnotetext{
*whhwung@mail.ncku.edu.tw
}

Published by the American Physical Society under the terms of the Creative Commons Attribution 4.0 International license. Further distribution of this work must maintain attribution to the author(s) and the published article's title, journal citation, and DOI. Funded by SCOAP. consider further generalizations. In the first part of this paper we extend the known butterfly velocity formula to the anisotropic spacetime. We will apply our formula to several brane systems. Our computations lead us to conjecture that the butterfly velocity is a quantity that is invariant under a double-dimensional reduction. In the second part of the paper, we consider the butterfly velocity for the GaussBonnet gravity with arbitrary matter fields. Butterfly velocity in the higher-derivative gravity, including the Gauss-Bonnet term without matter fields, has been discussed in [8] and [18]. The Gauss-Bonnet gravity is the simplest correction of the Einstein theory, without introducing derivatives higher than the second, appearing in the field equation. In the AdS/CFT correspondence, the Gauss-Bonnet term in the bulk corresponds to next-toleading order corrections in the $1 / \mathrm{N}$ expansion of the dual CFT [28]. Here we add the matter fields and apply our general formula to evaluate the butterfly velocity in several interesting holographic systems.

After reviewing the previous method, we extend the butterfly velocity formula $[8,10,11]$ to the anisotropic spacetime in Sec. II. We present a rather simple way, i.e., without too many complicated tensor calculations, to derive a general formula given in (2.30). In Sec. III we apply this formula to calculate the butterfly velocities in M2-branes, M5 branes, Dp-branes and also string backgrounds. We find that the butterfly velocity in M2 equals that in the string background. Also, the velocity in M5 equals that in D4. Since the spacetime of the string and D4 are equal to those through double-dimensional reduction from the M2 and M5 backgrounds, respectively, we conjecture that the butterfly velocity is invariant under a double-dimensional reduction. One more example involving the intersections $\mathrm{F} 1 \perp \mathrm{D} 4$ and $\mathrm{M} 2 \perp \mathrm{M} 5$, which are 
related also through a double-dimensional reduction, also supports our claim. In Sec. IV, we develop a butterfly velocity formula of Gauss-Bonnet gravity with arbitrary matter fields. We present the detailed tensor analysis and obtain a compact and general formula given in (4.18). For the special cases of planar, spherical, or hyperbolic black holes, the formula becomes a simpler form described in (4.22). In Sec. V we first check that our formula reproduces the previous result in the literature without matter fields $[8,18]$. We next apply our formula to the Einstein-GaussBonnet-Maxwell theory and Einstein-Gauss-Bonnet-scalar theory. Furthermore, we calculate the butterfly velocities in the Einstein-Gauss-Bonnet-Maxwell theory with spherical or hyperbolic black holes. We discuss future works in the last section. In Appendix A, we briefly describe the Kruskal coordinate in general geometry and derive some relations which are useful in computing butterfly velocity. In Appendix B, we present some details in deriving our formula of the butterfly velocity in Einstein-GaussBonnet gravity with arbitrary matter fields.

\section{SHOCK-WAVE EQUATION AND BUTTERFLY VELOCITY IN ANISOTROPIC SPACETIME}

\section{A. Shock-wave geometry and shock-wave equation}

We will derive the formula of butterfly velocity in the following anisotropic background:

$$
\begin{aligned}
d s^{2}= & -a(r) f(r) d t^{2}+\frac{d r^{2}}{b(r) f(r)} \\
& +\sum_{S=1}^{n} G_{i j}^{(S)}(r, x) d x_{(S)}^{i} d x_{(S)}^{j} \\
G_{i j}^{(S)}(r, x)= & h^{(S)}(r) g_{i j}^{(S)}(x),
\end{aligned}
$$

where horizon locates at $r=r_{H}$. Note $f\left(r_{H}\right)=0$ while $a\left(r_{H}\right) \neq 0, b\left(r_{H}\right) \neq 0$. In the original derivation [27], Sfetsos considered an isotropic background representing the special case $S=1$. The authors in [17,22-26] considered the anisotropic case in flat space where $g_{i j}^{(1)}=g_{i j}^{(2)}=\delta_{i j}$. In our study, the relevant line element [for example, $\left.g_{i j}^{(2)}(x) d x_{(2)}^{i} d x_{(2)}^{j}\right]$ will be able to describe curved surface where $g_{i j}^{(2)} \neq \delta_{i j}$. Note the associated temperature of the above black hole or black brane is given by

$$
T=\frac{f^{\prime}\left(r_{H}\right) \sqrt{a\left(r_{H}\right) b\left(r_{H}\right)}}{4 \pi} .
$$

Since the holographic geometry of the chaos covers two sides, we consider the line element expressed in the Kruskal coordinate: ${ }^{1}$

\footnotetext{
${ }^{1}$ In Appendix A, we describe more properties of the Kruskal coordinate. We also present several useful relations.
}

$$
\begin{aligned}
d s^{2}= & -\frac{4 a(r) f(r)}{a\left(r_{H}\right) b\left(r_{H}\right) f^{\prime}\left(r_{H}\right)^{2}} e^{-f^{\prime}\left(r_{H}\right) r_{*} \sqrt{a\left(r_{H}\right) b\left(r_{H}\right)}} d U d V \\
& +\sum_{S} G_{i j}^{(S)}(r, x) d x_{(S)}^{i} d x_{(S)}^{j} \\
= & 2 A(U, V) d U d V \\
& +\sum_{S} h^{(S)}(U V) g_{i j}^{(S)}(x) d x_{(S)}^{i} d x_{(S)}^{j} \\
U V= & e^{f^{\prime}\left(r_{H}\right) r_{*} \sqrt{a\left(r_{H}\right) b\left(r_{H}\right)}} \\
U / V= & e^{f^{\prime}\left(r_{H}\right) t \sqrt{a\left(r_{H}\right) b\left(r_{H}\right)}}
\end{aligned}
$$

in which $r_{*}$ is the tortoise coordinate defined by $d r_{*}=\frac{d r}{f(r) \sqrt{a(r) b(r)}}$.

To proceed we follow $[5,10,11,27]$ to add a small null perturbation of asymptotic energy $E$. At later times the perturbation will follow null trajectories very close to the (past) horizon, where the trajectories become exponentially blue-shifted and the perturbation grows exponentially large, in contrast to what was expected in an earlier study [29]. After solving the associated Einstein equation, one obtains the shock-wave geometry shown in Fig. 1.

We first describe the computation scheme of obtaining the shock-wave equation in spacetime (2.1). This will enable us to find butterfly velocities in M-branes, D-branes, and also string backgrounds. The scheme was clearly described in [27], and it has been applied to study the holographic butterfly velocity with matter fields in [10] recently.

The metric (2.1) describes the solution of the Einstein equation with a general form of stress tensor. We can express it as

$$
\begin{aligned}
G= & T_{\text {matter }} \\
= & 2 T_{U V}(U, V, x) d U d V+T_{U U}(U, V, x) d U d U \\
& +T_{V V}(U, V, x) d V d V+\sum_{S} T_{i j}^{(S)}(U, V, x) d x_{(S)}^{i} d x_{(S)}^{j},
\end{aligned}
$$

where $G$ is the Einstein tensor. Along the arguments of Dray and G. t'Hooft [29], for $U<0$ the spacetime is

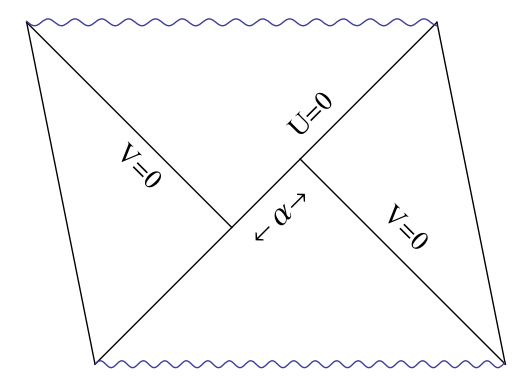

FIG. 1. Shock-wave geometry: Penrose diagram of an eternal black hole perturbed by a shock wave. 
described by (2.5). After adding a small null perturbation, for $U>0$ the spacetime is still described by (2.5), but $V$ is shifted by

$$
V \rightarrow V+\alpha(x)
$$

as shown in Fig. 1. The function $\alpha(x)$ will be determined by a shock-wave equation which is to be determined. The resulting metric and energy momentum tensor are

$$
\begin{aligned}
d s^{2}= & 2 A(U, V+\Theta \alpha(x)) d U\left(d V+\Theta \alpha^{\prime}(x) d x\right) \\
& +\sum_{S} G_{i j}^{(S)}(U,+\Theta \alpha(x), x) d x_{(S)}^{i} d x_{(S)}^{j} \\
T_{\text {matter }}= & 2 T_{\mathrm{UV}}(U, V+\Theta \alpha(x), x) d U\left(d V+\Theta \alpha^{\prime}(x) d x\right) \\
& +T_{U U}(U, V+\Theta \alpha(x), x) d U d U \\
& +T_{V V}(U, V+\Theta \alpha(x), x)\left(d V+\Theta \alpha^{\prime}(x) d x\right)^{2} \\
& +\sum_{S} T_{i j}^{(S)}(U, V+\Theta \alpha(x), x) d x_{(S)}^{i} d x_{(S)}^{j},
\end{aligned}
$$

where $\Theta=\Theta(U)$ is a step function and

$$
\alpha^{\prime}(x) d x=\sum_{i, S} \frac{\partial \alpha(x)}{\partial x_{(S)}^{i}} d x_{(S)}^{i} .
$$

As described by Sfetsos [27], in terms of the new coordinates

$$
\begin{gathered}
\hat{U}=U, \quad \hat{V}=V+\Theta \alpha(x), \\
\hat{x}_{(S)}^{i}=x_{(S)}^{i} \quad\left(\text { i.e. } \hat{X}_{\mu}=X_{\mu}\right),
\end{gathered}
$$

the metric and stress tensor can be expressed by the following simple forms:

$$
\begin{aligned}
d s^{2}= & 2 \hat{A}(\hat{U}, \hat{V}) d \hat{U} d \hat{V}+\sum_{S} \hat{G}_{i j}^{(S)}(\hat{U}, \hat{V}, \hat{x}) d \hat{x}_{(S)}^{i} d \hat{x}_{(S)}^{j} \\
& -2 \hat{A} \hat{\alpha}(\hat{x}) \hat{\delta}(\hat{U}) d \hat{U}^{2} \\
T_{\text {matter }}= & 2\left(\hat{T}_{\hat{U} \hat{V}}-T_{\hat{V} \hat{V}} \hat{\alpha} \hat{\delta}\right) d \hat{U} d \hat{V} \\
& +\left(T_{\hat{U} \hat{U}}+T_{\hat{V} \hat{V}} \hat{\alpha}^{2} \hat{\delta}^{2}-2 T_{\hat{U} \hat{V}} \hat{\alpha} \hat{\delta}\right) d \hat{U}^{2} \\
& +T_{\hat{V} \hat{V}} d \hat{V}^{2}+\sum_{S} \hat{T}_{i j}^{(S)} d \hat{x}_{(S)}^{i} d \hat{x}_{(S)}^{j} .
\end{aligned}
$$

Following [8,27], we add an extra stress tensor

$$
T_{(\text {shock }) \hat{U} \hat{U}}=E e^{\frac{2 \pi t}{\beta}} a(x) \delta(U)
$$

to produce the shock-wave geometry.

Now we have to solve the Einstein equation (we will drop the hat notation in what follows):

$$
G=T_{\text {matter }}+T_{\text {shock }} \text {. }
$$

The key point is that using the unperturbed Einstein equation $G_{V V}^{(0)}=T_{V V}^{(0)}$, then [21]

$$
T_{V V}^{(0)}=0,
$$

because $G_{V V}^{(0)}=0$ in the model spacetime. Therefore, $T_{\text {matter }}$ remains only one term that is linear in perturbation: $-2 T_{\hat{U} \hat{V}} \hat{\alpha} \hat{\delta} d \hat{U}^{2}$. The shock-wave equation can be written as

$$
G_{U U}^{(1)}+2 G_{\mathrm{UV}}^{(0)} \alpha(x) \delta(U)=C E e^{\frac{2 \pi t}{\beta}} a(x) \delta(U),
$$

where $G_{U U}^{(1)}$ is the first-order correction of the Einstein tensor from the metric (2.13).

\section{B. Butterfly velocity formula in anisotropic spacetime}

According to the scheme described above, the main problem is to calculate the tensors $G_{U U}^{(1)}$ and $G_{\mathrm{UV}}^{(0)}$. For the case of $S=1$, the above two tensors were presented in [27]. Here we are interested in a more general case.

We first need the following tensor properties in unperturbed spacetime (2.5):

$$
\begin{aligned}
G_{V V}^{(0)}(0)= & 0 \\
G_{\mathrm{UV}}^{(0)}= & -\frac{A^{\prime}(0)}{A(0)}-\sum_{S} \operatorname{dim}(S) \frac{h^{\prime(S)}(0)}{2 h^{(S)}(0)} \\
& -\frac{1}{2} A(0) R^{(0)} .
\end{aligned}
$$

They are found through straightforward algebra calculations. Note $\operatorname{dim}(S)=g^{(S) i j} g_{i j}^{(S)}$ arises from the tensor contraction from different species. We have considered values on the horizon, $U=0$.

In contrast to performing straightforward but tedious algebra calculations, we will calculate $G_{U U}^{(1)}$ starting from the following basic relation:

$$
\begin{aligned}
\delta R_{a b}= & \frac{1}{2}\left(\nabla^{c} \nabla_{a} \delta g_{c b}+\nabla^{c} \nabla_{b} \delta g_{c a}\right. \\
& \left.-g^{c d} \nabla_{a} \nabla_{b} \delta g_{c d}-\nabla^{2} \delta g_{a b}\right) .
\end{aligned}
$$

Now from $\delta g_{a b}=-2 A \alpha(x) \delta(U) \delta_{a U} \delta_{b U} \quad$ and $\quad g_{U U}^{(0)}=$ $g^{(0) U U}=0$ we find

$$
\begin{aligned}
G_{U U}^{(1)} & =\nabla^{\lambda} \nabla_{U} \delta g_{\lambda U}-\frac{1}{2} \nabla^{2} K-\frac{1}{2} K R^{(0)} \\
K & =-2 A(U V) \alpha(x) \delta(U) .
\end{aligned}
$$


Note that in the above relations the covariant derivative acts on unperturbed background. It is interesting that the term $\frac{1}{2} K R^{(0)}$ is canceled by $\frac{1}{2} A(0) R^{(0)}$ in (2.20), according to the shock-wave equation (2.18). To proceed we find

$$
\nabla^{2} K=\frac{1}{A \prod_{s} \sqrt{h^{(S)} g_{i j}^{(S)}}} \partial_{a}\left[\left(A \prod_{S} \sqrt{h^{(S)} g_{i j}^{(S)}}\right) g^{a b} \partial_{a} K\right]
$$

$$
\begin{aligned}
= & \left(\frac{4 A^{\prime}(0)}{A(0)}+\sum_{S} \frac{\operatorname{dim}(S) h^{\prime(S)}(0)}{h^{(S)}(0)}\right) \alpha(x) \delta(U) \\
& -2 A(0) \delta(U) \sum_{S} \frac{\Delta^{(S)} \alpha(x)}{h^{(S)}}
\end{aligned}
$$

$$
\nabla^{\lambda} \nabla_{U} \delta g_{\lambda U}=\left(\frac{2 A^{\prime}(0)}{A(0)}+\sum_{S} \operatorname{dim}(S) \frac{h^{\prime(S)}(0)}{h^{(S)}(0)}\right) \alpha(x) \delta(U),
$$

where $g^{a b}$ is the metric given in (2.5). The Laplacian is

$$
\Delta^{(S)} \alpha(x)=\frac{1}{\sqrt{g^{(S)}}} \partial_{j}^{(S)}\left(\sqrt{g^{(S)}} g^{(S) i j} \partial_{j}^{(S)} \alpha(x)\right) .
$$

To obtain above result we have used $\delta^{\prime}(U)=-\delta(U) / U$.

Collecting all the above results, we finally obtain

$$
\begin{aligned}
G_{U U}^{(1)}+2 G_{\mathrm{UV}}^{(0)} \alpha(x) \delta(U)= & \delta(U) \sum_{S} \frac{A(0)}{h^{(S)}(0)} \Delta^{(S)} \alpha(x) \\
& -\sum_{S} \operatorname{dim}(S) \frac{h^{\prime(S)}(0)}{2 h^{(S)}(0)} \alpha(x) \delta(U) .
\end{aligned}
$$

Note $R^{(0)}$ parts are canceled out. The shift function $\alpha(t, x)$ is determined by the perturbation function $a(x)$ through the shock wave equation

$$
\begin{aligned}
& {\left[A\left(U_{H}\right) \sum_{S}\left(h^{(S)}\right)^{-1} \Delta^{(S)}\right.} \\
& \left.\quad-\frac{1}{2} \sum_{S} G^{(S) i j}\left(U_{H}, x\right) G_{i j}^{(S)}\left(U_{H}, x\right)\right] \alpha(t, x)=E e^{2 \pi t / \beta} a(x),
\end{aligned}
$$

where $G_{i j}^{(S)}\left(U_{H}, x\right)=\left.\frac{\partial G_{i j}^{(S)}(u, x)}{\partial U}\right|_{U=U_{H}}$.

To obtain a simple formula of butterfly velocity we can consider the case in which the local source is $a(x)=\delta\left(x_{i}^{(Q)}\right)$. Using the relation (2.2) we find

$$
\begin{aligned}
& {\left[\Delta^{(Q)}-h^{(Q)} \sum_{S} \operatorname{dim}(S) \frac{h^{\prime(S)}}{2 A h^{(S)}}\right]_{U_{H}} \alpha\left(t, x_{i}^{(Q)}\right)} \\
& \quad=E e^{2 \pi t / \beta} \frac{h^{(Q)}\left(U_{H}\right)}{A\left(U_{H}\right)} \delta\left(x_{i}^{(Q)}\right),
\end{aligned}
$$

where $\operatorname{dim}(S)$ is the spatial dimension of $x_{(S)}^{i}$. The velocity along the direct $x_{(Q)}^{i}$ in the anisotropic spacetime (2.1) can be found to be

$$
\begin{aligned}
v_{B}^{(Q)} & =\frac{2 \pi k T}{M_{(Q)}} \\
M_{(Q)}^{2} & =h^{(Q)}\left(r_{H}\right) \sum_{S} \operatorname{dim}(S) \frac{b\left(r_{H}\right) f^{\prime}\left(r_{H}\right) h^{\prime(S)}\left(r_{H}\right)}{4 h^{(S)}\left(r_{H}\right)},
\end{aligned}
$$

where we have used

$$
\begin{aligned}
& h^{\prime}(U=0)=r_{H} h^{\prime}\left(r_{H}\right), \\
& A(U=0)=\frac{2 r_{H}}{b\left(r_{H}\right) f^{\prime}\left(r_{H}\right)},
\end{aligned}
$$

which are derived in Appendix A. Note that our formula (2.30) reduces to [17,22-26] and [27] in the flat anisotropic space and curved isotropic space, respectively. In the next section we will use our new formulas to evaluate the butterfly velocities in the brane geometry.

\section{BUTTERFLY VELOCITY IN BRANE GEOMETRY}

\section{A. M5 and D4 backgrounds}

In this section we use our general butterfly velocity formula to calculate the butterfly velocities in M-branes, D-branes, and string backgrounds. The $N_{5}$ black M5branes solution is given by [30,31]

$$
\begin{aligned}
d s_{M 5}^{2}= & H^{\frac{-1}{3}}\left(-f(r) d t^{2}+d x_{1}^{2}+d x_{2}^{2}+d x_{3}^{2}+d x_{4}^{2}+d x_{5}^{2}\right) \\
& +H^{\frac{2}{3}}\left(\frac{d r^{2}}{f(r)}+r^{2} d \Omega_{4}^{2}\right)
\end{aligned}
$$

where $H$ is the harmonic function defined by

$$
H=1+\frac{N_{5}}{r^{3}} .
$$

The function $f(r)$ specified by the horizon at $r_{H}$ is

$$
f(r)=1-\frac{r_{H}^{3}}{r^{3}}
$$

We can approximate $H \sim \frac{N_{5}}{r^{3}}$ in the "near-horizon" limit. On the other hand, the spacetime of a stack of $N_{4}$ black D4-branes (in the Einstein frame) is given by 
TABLE I. Parameter functions for M5 and D4.

\begin{tabular}{lccccccc}
\hline \hline & $a\left(r_{H}\right)$ & $b\left(r_{H}\right)$ & $h_{M}\left(r_{H}\right)$ & $\operatorname{dlim}(\mathrm{M})$ & $h_{\Omega}\left(r_{H}\right)$ & $\operatorname{dim}(\Omega)$ & $f^{\prime}\left(r_{H}\right)$ \\
\hline M5 & $\frac{r_{H}}{N_{5}^{1 / 3}}$ & $\left(\frac{r_{H}}{N_{5}^{1 / 3}}\right)^{2}$ & $\frac{r_{H}}{N_{5}^{1 / 3}}$ & 5 & $r_{H}^{2}\left(\frac{r_{H}}{N_{5}^{1 / 3}}\right)^{-2}$ & 4 & $\frac{3}{r_{H}}$ \\
D4 & $\left(\frac{r_{H}}{N_{4}^{1 / 3}}\right)^{9 / 8}$ & $\left(\frac{r_{H}}{N_{4}^{1 / 3}}\right)^{15 / 8}$ & $\left(\frac{r_{H}}{N_{4}^{1 / 3}}\right)^{9 / 8}$ & 4 & $r_{H}^{2}\left(\frac{r_{H}}{N_{4}^{1 / 3}}\right)^{-15 / 8}$ & 4 & $\frac{3}{r_{H}}$ \\
\hline \hline
\end{tabular}

$$
\begin{aligned}
d s_{D 4}^{2}= & -H^{\frac{-3}{8}} f(r) d t^{2}+H^{\frac{-3}{8}}\left(d x_{1}^{2}+d x_{2}^{2}+d x_{3}^{2}+d x_{4}^{2}\right) \\
& +H^{\frac{5}{8}}\left(\frac{d r^{2}}{f(r)}+r^{2} d \Omega_{4}^{2}\right) \\
H= & 1+\frac{N_{4}}{r^{3}}, \quad f(r)=1-\frac{r_{H}^{3}}{r^{3}} .
\end{aligned}
$$

In the "near-horizon" limit, $H \sim \frac{N_{4}}{r^{3}}$. Using these metrics, we calculate the black-brane temperature, $T\left(r_{H}\right)$, the butterfly velocities $v_{B}$ along $\left(x_{1} \cdots x_{5}\right)$ and along $\Omega_{4}$ in M5. We can also compute the velocities along $\left(x_{1} \cdots x_{4}\right)$ and along $\Omega_{4}$ in D4. We collect the relevant functions in Table 1.

It seems striking that although the parameter functions for M5 and D4 are quite different, the resulting butterfly velocities and temperature, given below,

$$
\begin{array}{cccc} 
& T\left(r_{H}\right) & v_{B}(M) & v_{B}(\Omega) \\
M 5 & \frac{3}{4 \pi} \sqrt{\frac{r_{H}}{N_{5}}} & \sqrt{\frac{3}{5}} & \frac{4 \pi}{\sqrt{15}} T \\
\text { D4 } & \frac{3}{4 \pi} \sqrt{\frac{r_{H}}{N_{4}}} & \sqrt{\frac{3}{5}} & \frac{4 \pi}{\sqrt{15}} T,
\end{array}
$$

are the same. Let us make following comments:

(1) For temperature to be the same, one needs the same number of branes for M5 and D4. The butterfly velocities are the same independent of $\mathrm{N}$.

(2) While the butterfly velocity $v_{B}(M)$ satisfies the upper bound, $v_{B}(\Omega)$ violates it. This violation does not contract to [4] since that paper assumes space isotropy $[15,17]$.

(3) Notice that through a double-dimensional reduction M5 becomes D4.

(4) For completeness we present below the butterfly velocity in the Dp brane background:

$$
\begin{array}{cccc} 
& T\left(r_{H}\right) & v_{B}(M) & v_{B}(\Omega) \\
D p & \frac{7-p}{4 \pi \sqrt{N p}} r_{H}^{\frac{5-p}{2}} & \sqrt{\frac{7-p}{9-p}} & \frac{4 \pi T}{\sqrt{(7-p)(9-p)}}
\end{array} .
$$

\section{B. M2 and fundamental string (F1) backgrounds}

Now we consider the systems of M2 and fundamental string (F1) backgrounds. The relevant functions are collected in Table 2.

We again observe that although the parameter functions for $\mathrm{M} 2$ and $\mathrm{F} 1$ are different, the temperature and butterfly velocities, shown below,

$$
\begin{array}{cccc} 
& T\left(r_{H}\right) & v_{B}(M) & v_{B}(\Omega) \\
M 2 & \frac{3 r_{H}^{2}}{2 \pi \sqrt{N_{2}}} & \frac{\sqrt{3}}{2} & \frac{\pi}{\sqrt{3}} T, \\
F 1 & \frac{3 r_{H}}{2 \pi \sqrt{F_{1}}} & \frac{\sqrt{3}}{2} & \frac{\pi}{\sqrt{3}} T
\end{array}
$$

have the same behavior. Since that through a doubledimensional reduction M2 (M5) becomes F1 (D4) one tempts to conjecture that the butterfly velocity is generally invariant under a double-dimensional reduction. Let us consider one more example in the next section.

\section{Intersections M2 $\perp$ M5 and F1 $\perp$ D4 backgrounds}

We consider the brane intersection $\mathrm{M} 2 \perp \mathrm{M} 5$ system where M2-branes lie in $(w, x)$ and M5-branes locate at $\left(w, y_{1} \ldots y_{4}\right)$, i.e.,

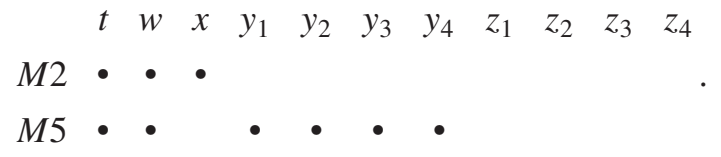

Upon a double-dimensional reduction, the space $w$ is wrapped, and the geometry becomes the intersection $\mathrm{F} 1 \perp \mathrm{D} 4$, i.e.,

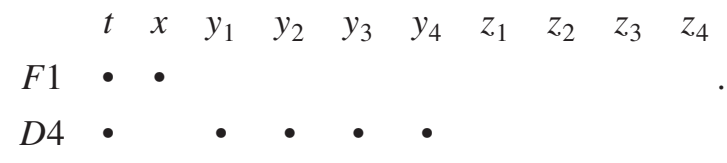

The metric of black intersection $\mathrm{M} 2 \perp \mathrm{M} 5$ is $[30,31]$

TABLE II. Parameter functions for M2 and F1.

\begin{tabular}{lccccccc}
\hline \hline & $a\left(r_{H}\right)$ & $b\left(r_{H}\right)$ & $h_{M}\left(r_{H}\right)$ & $\operatorname{dim}(\mathrm{M})$ & $h_{\Omega}\left(r_{H}\right)$ & $\operatorname{dim}(\Omega)$ & $f^{\prime}\left(r_{H}\right)$ \\
\hline M2 & $\left(\frac{r_{H}}{N_{2}^{1 / 6}}\right)^{4}$ & $\left(\frac{r_{H}}{N_{2}^{1 / 6}}\right)^{2}$ & $\left(\frac{r_{H}}{N_{2}^{1 / 6}}\right)^{4}$ & 2 & $r_{H}^{2}\left(\frac{r_{H}}{N_{2}^{1 / 6}}\right)^{-2}$ & 7 & $\frac{6}{r_{H}}$ \\
F1 & $\left(\frac{r_{H}}{F 1^{1 / 6}}\right)^{9 / 2}$ & $\left(\frac{r_{H}}{F 1^{1 / 6}}\right)^{3 / 2}$ & $\left(\frac{r_{H}}{F 1^{1 / 6}}\right)^{9 / 2}$ & 1 & $r_{H}^{2}\left(\frac{r_{H}}{F 1^{1 / 6}}\right)^{-3 / 2}$ & 7 & $\frac{6}{r_{H}}$ \\
\hline \hline
\end{tabular}


TABLE III. Parameter functions for M2 $\perp$ M5 and F1 $\perp$ D4.

\begin{tabular}{|c|c|c|c|c|c|c|}
\hline & $a\left(r_{H}\right)$ & $b\left(r_{H}\right)$ & $h_{w}\left(r_{H}\right)$ & $\operatorname{dim}(w)$ & $h_{x}\left(r_{H}\right)$ & $\operatorname{dim}(x)$ \\
\hline$\overline{\mathrm{M} 2 \perp \mathrm{M} 5}$ & $\left(\frac{r_{H}^{2}}{H}\right)^{2 / 3}\left(\frac{r_{H}^{2}}{N}\right)^{1 / 3}$ & $\left(\frac{r_{H}^{2}}{N}\right)^{1 / 3}\left(\frac{r_{H}^{2}}{N}\right)^{2 / 3}$ & $\left(\frac{r_{H}^{2}}{N}\right)^{2 / 3}\left(\frac{r_{H}^{2}}{N)_{0}}\right)^{1 / 3}$ & 1 & $\left(\frac{r_{H}^{2}}{N}\right)^{2 / 3}\left(\frac{r_{H}^{2}}{N}\right)^{-2 / 3}$ & 1 \\
\hline $\mathrm{F} 1 \perp \mathrm{D} 4$ & $\left(\frac{r_{H}^{2}}{F_{1}}\right)^{3 / 4}\left(\frac{r_{H}^{2}}{N_{4}}\right)^{3 / 8}$ & $\left(\frac{r_{H}^{2}}{F_{1}}\right)^{1 / 4}\left(\frac{r_{H}^{2}}{N_{4}}\right)^{5 / 8}$ & wrapped & 0 & $\left(\frac{r_{H}^{2}}{F_{1}}\right)^{3 / 4}\left(\frac{r_{H}^{2}}{N_{4}}\right)^{-5 / 8}$ & 1 \\
\hline & $h_{y}\left(r_{H}\right)$ & $\operatorname{dim}(y)$ & \multicolumn{2}{|c|}{$h_{\Omega}\left(r_{H}\right)$} & $\operatorname{dim}(\Omega)$ & $f^{\prime}\left(r_{H}\right)$ \\
\hline$\overline{\mathrm{M} 2 \perp \mathrm{M} 5}$ & $\left(\frac{r_{H}^{2}}{N_{2}}\right)^{-1 / 3}\left(\frac{r_{H}^{2}}{N_{s}}\right)^{1 / 3}$ & 4 & \multicolumn{2}{|c|}{$r_{2}^{H}\left(\frac{r_{H}^{2}}{N_{2}}\right)^{-1 / 3}\left(\frac{r_{H}^{2}}{N_{5}}\right)^{-2 / 3}$} & 3 & $\frac{2}{r_{H}}$ \\
\hline $\mathrm{F} 1 \perp \mathrm{D} 4$ & $\left(\frac{r_{H}^{2}}{F_{F}}\right)^{-1 / 4}\left(\frac{r_{H}^{2}}{N_{H}}\right)^{3 / 8}$ & 4 & \multicolumn{2}{|c|}{$r_{2}^{H}\left(\frac{r_{H}^{2}}{F_{1}}\right)^{-1 / 4}\left(\frac{r_{H}^{2}}{N_{4}}\right)^{-5 / 8}$} & 3 & $\frac{2}{r_{H}}$ \\
\hline
\end{tabular}

$$
\begin{aligned}
d s_{M 2 \perp M 5}^{2}= & H_{2}^{\frac{-2}{3}} H_{5}^{\frac{-1}{3}}\left[\left(-f(r) d t^{2}+d w^{2}\right]+H_{2}^{\frac{-2}{3}} H_{5}^{\frac{2}{3}} d x^{2}\right. \\
& +H_{2}^{\frac{1}{3}} H_{5}^{\frac{-1}{3}} d \vec{y}^{2}+H_{2}^{\frac{1}{3}} H_{5}^{\frac{2}{3}}\left[f^{-1}(r) d r^{2}+r^{2} d \Omega_{3}^{2}\right],
\end{aligned}
$$

where

$H_{2}=1+\frac{N_{2}}{r^{2}}, \quad H_{5}=1+\frac{N_{5}}{r^{2}}, \quad f(r)=1-\frac{r_{H}^{2}}{r^{2}}$.

In the Einstein frame, the metric of the intersection $\mathrm{F} 1 \perp \mathrm{D} 4$ is

$$
\begin{aligned}
d s_{F 1 \perp D 4}^{2}= & -H_{1}^{\frac{-3}{4}} H_{4}^{\frac{-3}{8}} f(r) d t^{2}+H_{1}^{\frac{-3}{4}} H_{4}^{\frac{5}{8}} d x^{2}+H_{1}^{\frac{1}{4}} H_{4}^{\frac{-3}{8}} d \vec{y}^{2} \\
& +H_{1}^{\frac{1}{4}} H_{4}^{\frac{5}{8}}\left[f^{-1}(r) d r^{2}+r^{2} d \Omega_{3}^{2}\right],
\end{aligned}
$$

where

$H_{1}=1+\frac{F_{1}}{r^{2}}, \quad H_{4}=1+\frac{N_{4}}{r^{2}}, \quad f(r)=1-\frac{r_{H}^{2}}{r^{2}}$.

In the "near-horizon" limit, the relevant functions are collected in Table 3.

Although the parameter functions for $\mathrm{M} 2 \perp \mathrm{M} 5$ and F1 $\perp D 4$ are different, the temperature and butterfly velocities,

$$
\begin{array}{ccccc}
\square & T\left(r_{H}\right) & v_{B}(x) & v_{B}(y) & v_{B}(\Omega) \\
M 2 \perp M 5 & \frac{r_{H}}{2 \pi \sqrt{N_{2} N_{5}}} & 2 \pi T \sqrt{N_{2}} & 2 \pi T \sqrt{N_{5}} & 2 \pi T \\
F 1 \perp D 4 & \frac{r_{H}}{2 \pi \sqrt{F_{1} N_{4}}} & 2 \pi T \sqrt{F_{1}} & 2 \pi T \sqrt{N_{4}} & 2 \pi T
\end{array}
$$

again have the same behaviors. Thus, the butterfly velocity is again invariant under a double-dimensional reduction.

\section{FORMULA OF BUTTERFLY VELOCITY IN EINSTEIN-GAUSS-BONNET GRAVITY WITH MATTER FIELDS}

\section{A. Formula in arbitrary spacetime}

The Lagrangian we consider will contain a curvature scalar, a cosmological constant, a Gauss-Bonnet term, and matter fields:

$$
\begin{aligned}
\mathcal{L}= & R+\frac{d(d+1)}{\ell_{\text {AdS }}^{2}}+\frac{\gamma_{G B}}{2}\left(R_{a b c d} R^{a b c d}-4 R_{a b} R^{a b}+R^{2}\right) \\
& +\mathcal{L}_{\text {matter }},
\end{aligned}
$$

where $\ell_{\text {AdS }}$ is the AdS radius. The associated gravity equation is given by

$G_{a b}+\frac{2 d(d+1)}{\ell_{\mathrm{AdS}}^{2}} g_{a b}-\gamma_{G B}\left(H_{a b}-\frac{1}{4} H g_{a b}\right)=T_{a b}$,

where we denote

$H_{a b}=R_{a c d e} R_{b}^{c d e}-2 R_{a c b d} R^{c d}-2 R_{a c} R_{b}^{c}+R_{a b} R$.

The Gauss-Bonnet term is a special combination of curvatures in which its field question contains only second-order derivatives. All higher-derivative terms are canceled out. In four-dimensions, the Gauss-Bonnet term is a topological invariant so it does not enter dynamics. We will consider the theory in $d+2$ dimensions. ${ }^{2}$ We assume that the solution expressed in Kruskal coordinates could be written as

$d s^{2}=2 A(U, V) d U d V+h(U, V) \sum_{i, j=1}^{d} g_{i j}(x) d x^{i} d x^{j}$.

The above metric leads to

$$
H_{V V}^{(0)}-\frac{1}{4} H^{(0)} g_{V V}^{(0)}=0
$$

and the condition $T_{V V}^{(0)}=0$ is automatically satisfied. After perturbing the metric, we have an extra contribution on the $g_{U U}^{(1)}$ component, which is linear in $\alpha$ as described in (2.13).

In Gauss-Bonnet gravity, according to the scheme described in Sec. II, the shock-wave equation becomes

\footnotetext{
${ }^{2}$ Note that the total dimension of spacetime D in [8] and [18] is $\mathrm{D}=1+\mathrm{d}$, while in here we let $\mathrm{D}=2+\mathrm{d}$. In our notation the Laplacian is then denoted as $\Delta^{(d)}$ which is more convenient in our discussion.
} 


$$
\begin{aligned}
& {\left[H_{U U}^{(1)}-\frac{1}{4} H^{(0)} g_{U U}^{(1)}\right]+2\left[H_{\mathrm{UV}}^{(0)}-\frac{1}{4} g_{\mathrm{UV}}^{(0)} H^{(0)}\right] \alpha(x) \delta(U)} \\
& \quad=E^{2 \pi t / \beta} a(x) \delta(U)
\end{aligned}
$$

Using the definitions $g_{U U}^{(1)}=-2 A \alpha(x) \delta(U)$ and $g_{\mathrm{UV}}^{(0)}=A$, the second terms in the first bracket and second bracket are canceled exactly. This property is the same as what happens in Einstein gravity where the terms $\frac{1}{2} g_{\mathrm{UV}}^{(0)} R^{(0)}$ are canceled out in Sec. II. The shock-wave equation for Gauss-Bonnet gravity now has a very simple form:

$$
H_{U U}^{(1)}+2 H_{\mathrm{UV}}^{(0)} \alpha(x) \delta(U)=E^{2 \pi t / \beta} a(x) \delta(U) .
$$

Now we only have to calculate $H_{U U}^{(1)}$ and $H_{\mathrm{UV}}^{(0)}$, just as we only had to calculate $G_{U U}^{(1)}$ and $G_{\mathrm{UV}}^{(0)}$ in (2.13) for the Einstein gravity theory.

To proceed, we find the following general relations:

$$
\begin{aligned}
H_{\mathrm{UV}}^{(0)}= & -R^{(0)}\left[\frac{A^{\prime}}{A}+\frac{(d-2) h^{\prime}}{2 h}\right]-\frac{2 A^{\prime 2}}{A^{3}}-\frac{(3 d-2) A^{\prime} h^{\prime}}{A^{2} h} \\
& -\frac{d(d-3) h^{\prime 2}}{2 A h^{2}} \\
H_{U U}^{(1)}= & \left(R_{U c d e} R_{U}{ }^{c d e}\right)^{(1)}-2\left(R_{U c U d} R^{c d}\right)^{(1)} \\
& -2\left(R_{U c} R_{U}^{c}\right)^{(1)}+\left(R_{U U} R\right)^{(1)}
\end{aligned}
$$

where, up to a factor $\delta(U)$,

$$
\begin{aligned}
&\left(R_{U c d e} R_{U}{ }^{c d e}\right)^{(1)}=-\frac{4 A^{\prime 2}}{A^{3}} \alpha-\frac{2 h^{\prime}}{h^{2}} \Delta^{(d)} \alpha \\
&\left(R_{U c U d} R^{c d}\right)^{(1)}= A R^{(0) i j} \nabla_{i}^{(d)} \nabla_{j}^{(d)} \alpha(x)+\frac{A^{\prime}}{A h} \Delta^{(d)} \alpha(x) \\
&+\left(\frac{R^{(0)} h^{\prime}}{2 h}-\frac{2 A^{\prime 2}}{A^{3}}+\frac{d h^{\prime 2}}{A h^{2}}-\frac{(3 d-2) A^{\prime} h^{\prime}}{2 A^{2} h}\right) \\
& \times \alpha(x) \\
&\left(R_{U c} R_{U}^{c}\right)^{(1)}=-\left(\frac{2 A^{\prime 2}}{A^{3}}+\frac{d A^{\prime} h^{\prime}}{A^{2} h}\right) \alpha-\left(\frac{2 A^{\prime}}{A h}+\frac{d h^{\prime}}{h^{2}}\right) \Delta^{(d)} \alpha \\
&\left(R_{U U} R\right)^{(1)}=\left(\frac{2 A^{\prime}}{A}+\frac{d h^{\prime}}{2 h}\right) R^{(0)} \alpha+\frac{A}{h} R^{(0)} \Delta^{(d)} \alpha .
\end{aligned}
$$

The covariant derivative $\nabla_{i}^{(d)}$ and Laplacian $\Delta^{(d)}$ are defined in the d-dimensional metric, $d s^{2}=g_{i j} d x^{i} d x^{j}$. $R^{(0)}$ is the $2+\mathrm{d}$ dimensional Ricci scalar calculated by unperturbative metrics (4.4). Note that all values are evaluated at the horizon. It will require further analysis to derive the relation $\left(R_{U c U d} R^{c d}\right)^{(1)}$, which we present in
Appendix B. It is interesting to see that in the Einstein gravity the term containing derivatives of $\alpha(x)$ are formed as the Laplacian, $\Delta^{(d)} \alpha(x)$, while in the Gauss-Bonnet gravity it appears as a new form $R^{i j} \nabla_{i}^{(d)} \nabla_{j}^{(d)} \alpha(x)$.

Collect the above calculations, the shock-wave equation of Einstein-Gauss-Bonnet gravity from

$$
\begin{aligned}
& {\left[G_{U U}^{(1)}+2 G_{\mathrm{UV}}^{(0)} \alpha(x) \delta(U)\right]-\gamma_{G B}\left[H_{U U}^{(1)}+2 H_{\mathrm{UV}}^{(0)} \alpha(x) \delta(U)\right]} \\
& \quad=E e^{2 \pi t / \beta} a(x) \delta(U)
\end{aligned}
$$

reduces to the following formula:

$$
\begin{aligned}
& {\left[\Delta^{(d)} \alpha(x)-\frac{d h^{\prime}}{2 A} \alpha(x)\right]_{U=0}} \\
& \quad-\gamma_{G B}\left[-2 h R^{(0) i j} \nabla_{i}^{(d)} \nabla_{j}^{(d)} \alpha(x)\right. \\
& \quad+\left(R^{(0)}+\frac{2 A^{\prime}}{A^{2}}+\frac{2(d-1) h^{\prime}}{A h}\right) \Delta^{(d)} \alpha(x) \\
& \left.\quad-\left(\frac{R^{(0)}(d-2) h^{\prime}}{2 A}+\frac{(d-2) A^{\prime} h^{\prime}}{A^{3}}+\frac{d(d-2) h^{2}}{A^{2} h}\right) \alpha(x)\right]_{U=0} \\
& =\frac{h(0)}{A(0)} E e^{\frac{2 \pi t}{\beta}} a(x),
\end{aligned}
$$

evaluated in Kruskal coordinate, i.e., $A=A(U V)$ and $h=h(U V)$. Using the tensor relations

$$
\begin{gathered}
R^{(0) i j} \nabla_{i}^{(d)} \nabla_{j}^{(d)} \alpha(x)=\frac{1}{h^{2}} R^{(d) i j} \nabla_{i}^{(d)} \nabla_{j}^{(d)} \alpha(x)-\frac{h^{\prime}}{A h^{2}} \Delta^{(d)} \alpha(x) \\
R^{(0)}=\frac{R^{(d)}}{h}-\frac{2 A^{\prime}}{A^{2}}-\frac{2 d h^{\prime}}{A h}
\end{gathered}
$$

the butterfly velocity formula has a simple form

$$
\begin{gathered}
{\left[\Delta^{(d)} \alpha(x)-\frac{d h^{\prime}}{2 A} \alpha(x)\right]_{U=0}} \\
-\gamma_{G B}\left[-\frac{2 R^{(d) i j}}{h} \nabla_{i}^{(d)} \nabla_{j}^{(d)} \alpha(x)+\frac{R^{(d)}}{h} \Delta^{(d)} \alpha(x)\right. \\
\left.-\frac{(d-2) h^{\prime} R^{(d)}}{2 A h} \alpha(x)\right]_{U=0}=\frac{h(0)}{A(0)} E e^{\frac{2 \pi t}{\beta}} a(x),
\end{gathered}
$$

in which $R^{(d) i j}$ and $R^{(d)}$ are defined in the d-dimensional metric $d s^{2}=g_{i j} d x^{i} d x^{j}$.

\section{B. Formula in planar, spherical, and hyperbolic black holes}

To further simplify (4.18), let us consider $2+\mathrm{d}$ dimensional planar, spherical, or hyperbolic black holes. The general metric is 


$$
g_{i j}(x) d x^{i} d x^{j}=\left\{\begin{array}{cc}
d \theta_{1}^{2}+d \theta_{2}^{2}+\cdots+d \theta_{d}^{2}, & k=0 \\
d \theta_{1}^{2}+\sin ^{2} \theta_{1}\left(d \theta_{2}^{2}+\sin ^{2} \theta_{2}\left(d \theta_{3}^{2}+\cdots+\sin ^{2} \theta_{d-1} d \theta_{d}^{2}\right),\right. & k=1 \\
d \theta_{1}^{2}+\sinh ^{2} \theta_{1}\left(d \theta_{2}^{2}+\sin ^{2} \theta_{2}\left(d \theta_{3}^{2}+\cdots+\sin ^{2} \theta_{d-1} d \theta_{d}^{2}\right),\right. & k=-1,
\end{array}\right.
$$

then

$$
\begin{aligned}
R^{(d) i j} \nabla_{i}^{(d)} \nabla_{j}^{(d)} \alpha(x) & =k(d-1) \Delta^{(d)} \alpha(x) \\
R^{(d)} & =k d(d-1) .
\end{aligned}
$$

Substituting these relations into (4.18) and with the help of Appendix A, we obtain an amazingly simple expression for the shock-wave equation of Einstein-Gauss-Bonnet gravity with arbitrary matters:

$$
\left(1-k \frac{(d-2)(d-1) \gamma_{G B}}{h\left(r_{H}\right)}\right)\left(\Delta^{(d)}-M^{2}\right) \alpha(x) \sim \delta(x),
$$

where

$$
\begin{gathered}
M^{2}=\frac{d h^{\prime}(0)}{2 A(0)}=d \pi h^{\prime}\left(r_{H}\right) T \\
v_{B}=\frac{2 \pi T}{M}=\sqrt{\frac{4 \pi T}{d h^{\prime}\left(r_{H}\right)}} .
\end{gathered}
$$

Let us summarize and make some remarks.

(1) The shock-wave equation (4.18) can be directly applied to Einstein-Gauss-Bonnet theory with arbitrary matters with the black hole metric being expressed as (4.4). For planar, spherical, or hyperbolic black holes we have a simple formula (4.22).

(2) The case of $k=0$ was studied in [8].

(3) The time for the function $\alpha(t, x)$ becomes order-one after the perturbation is defined as the "screaming time". Using the function in (1.1) we see that $\alpha(t, x) \sim e^{\lambda\left(t-t *-\frac{|x|}{v_{B}}\right)}$. The formula (4.22) shows an overall factor which may be regarded as rescaling the perturbation source, or, effectively, be absorbed into the exponential in (1.1). This then changes the screaming time by the amount $\log \left(1-k \frac{(d-2)(d-1) \gamma_{G B}}{r_{H}^{2}}\right)$ as first found in [8].

(4) The factor $(d-2)(d-1) \gamma_{G B}$ vanishes for $d=1,2$. The reason is that: (a) The case of $d=1$ is in fact planar and it shall be zero. (b) The Gauss-Bonnet is topological if $d=2$, and zero value at $d=2$ reveals this property.

In the next section we consider some examples to illustrate our formulas.

\section{BUTTERFLY VELOCITY IN EINSTEIN-GAUSS-BONNET GRAVITY WITH MATTERS}

\section{A. Planar black hole}

In this subsection we consider the case in which the butterfly velocity is propagating in the planar black hole background.

\section{Einstein-Gauss-Bonnet gravity}

Consider first the simplest theory without any matter. The planar black hole solution is [32-34]

$d s^{2}=-f(r) N_{\sharp}^{2} d t^{2}+f(r)^{-1} d r^{2}+r^{2} \sum_{i=1}^{d} d x^{i} d x^{i}$,

with

$$
\begin{aligned}
f(r) & =\frac{r^{2}}{2 \lambda}\left[1-\sqrt{1-4 \lambda\left(1-\left(r / r_{H}\right)^{-d-1}\right)}\right] \\
T & =N_{\sharp}^{2} \frac{(d+1) r_{H}}{4 \pi} \\
N_{\sharp}^{2} & =\frac{1}{2}(1+\sqrt{1-4 \lambda}), \quad \lambda \equiv \gamma_{G B}(d-1)(d-2) .
\end{aligned}
$$

We let the AdS radius $\ell_{\text {AdS }}=1$. The horizon locates on $r_{H}$. As discussed in [8], the presence of the parameter $N_{\sharp}$ in the metric rescales time so the temperature is $\beta=4 \pi f^{\prime}(1) / N_{\sharp}$. This rescaling implies that the butterfly velocities in Einstein gravity and Einstein-Gauss-Bonnet gravity without matters are

$$
\begin{aligned}
v_{B}(0) & =\sqrt{\frac{(d+1)}{2 d}} \\
v_{B}\left(\gamma_{G B}\right) & =N_{\sharp} v_{B}(0) \\
& =\left(1+\sqrt{1-4(d-1)(d-2) \gamma_{G B}}\right)^{1 / 2} v_{B}(0),
\end{aligned}
$$

respectively. These results were first found in [8].

\section{Einstein-Gauss-Bonnet-Maxwell gravity}

For the Einstein-Gauss-Bonnet gravity theory with a Maxwell field, one adds a matter Lagrangian 


$$
\mathcal{L}_{\text {matter }}=-\frac{1}{4 g^{2}} F_{\mu \nu} F^{\mu \nu} .
$$

The associated planar black hole solution then is [32-34]

$$
\begin{aligned}
d s^{2} & =-f_{q}(r) N_{\sharp}^{2} d t^{2}+f_{q}(r)^{-1} d r^{2}+r^{2} \sum_{i=1}^{d} d x^{i} d x^{i} \\
A_{t} & =\mu\left(1-\left(r / r_{H}\right)^{-(d-1)}\right),
\end{aligned}
$$

where

$$
\begin{aligned}
f_{q}(r) & =\frac{r^{2}}{2 \lambda}\left[1-\sqrt{1-4 \lambda\left(1-(1+q)\left(r / r_{H}\right)^{-d-1}+q\left(r / r_{H}\right)^{-2 d}\right)}\right] \\
T & =N_{\sharp}^{2} \frac{((d+1)+(1-d) q) r_{H}}{4 \pi} \\
q & =\frac{d-1}{d} \frac{\mu^{2}}{g^{2} r_{H}^{2}} .
\end{aligned}
$$

The parameters $q$ and $\mu$ denote the strength of Maxwell fields. The values of $N_{\sharp}$ and $\lambda$ are defined before.

We find the butterfly velocities in the Einstein-Maxwell gravity theory and Einstein-Gauss-Bonnet-Maxwell gravity theory are given by

$$
\begin{aligned}
v_{B}^{(q)}(0) & =\sqrt{\frac{(d+1)+(1-d) q}{2 d}} \\
v_{B}^{(q)}\left(\gamma_{G B}\right) & =N_{\sharp} v_{B}^{(q)}(0) \\
& =\left(1+\sqrt{1-4(d-1)(d-2) \gamma_{G B}}\right)^{1 / 2} v_{B}^{(q)}(0),
\end{aligned}
$$

respectively. When $q=0$ above, results reduce to the previous case. Comparing (5.5) and (5.14) we observe that the ratio of butterfly velocities between those with and without the Gauss-Bonnet term is in fact "universal", in the sense that it does not depend on the strength of the Maxwell field.

\section{Einstein-Gauss-Bonnet-scalar gravity}

Consider the Einstein-Gauss-Bonnet gravity theory with scalar fields. The matter field Lagrangian is

$$
\mathcal{L}_{\text {matter }}=-\frac{1}{2} \partial_{\mu} \Phi \partial^{\mu} \Phi+\frac{\xi}{2} R \Phi+U(\Phi) .
$$

Choosing the particular values of parameters

$$
\begin{aligned}
\gamma_{G M} & =\frac{1}{2(d-1)(d-2)}, \quad \Lambda=-\frac{d(d+1)}{4} \\
\xi & =\frac{d}{4\left(d_{2}\right)}, \quad U(\Phi)=\frac{d^{2}}{32} \Phi^{2},
\end{aligned}
$$

where $\Lambda$ is the cosmological constant, the exact black hole solution can be described by $[35,36]$

$$
\begin{aligned}
& d s^{2}=-f(r) d t^{2}+f^{-1} d r^{2}+r^{2} \sum_{i=1}^{d} d x^{i} d x^{i} \\
& f(r)=r^{2}\left(1-\left(r / r_{H}\right)^{\frac{-d}{2}}\right), \quad T=\frac{r_{H} d}{8 \pi} .
\end{aligned}
$$

We find the butterfly velocity is

$$
v_{B}=\frac{1}{2} .
$$

Note that in this model the coupling of Gauss-Bonnet term $\gamma_{G B}$ is fixed in (5.16).

\section{B. Spherical and hyperbolic black holes}

Next we consider nonplanar black hole backgrounds, including spherical and hyperbolic geometry.

\section{Einstein gravity}

Consider first the simplest case. The metric of a black hole in Einstein gravity theory is 


$$
\begin{aligned}
d s^{2} & =-f(r) d t^{2}+f_{q}(r)^{-1} d r^{2}+r^{2} \sum_{i, j=1}^{d} g_{i j} d x^{i} d x^{j} \\
f(r) & =k+r^{2}-\left(\frac{r}{r_{H}}\right)^{1-d}\left(k+r_{H}^{2}\right) \\
T & =\frac{(d+1) r_{H}^{2}+(d-1) k}{4 \pi r_{H}}
\end{aligned}
$$

where $k=0,1,-1$ describes flat, spherical, and hyperbolic black holes, respectively. The relation between the horizon radius and the black hole temperature is more complicated, and is given by

$$
r_{H}=\frac{2 \pi T+\sqrt{4 \pi^{2} T^{2}+k\left(1-d^{2}\right)}}{(1+d)} .
$$

The butterfly velocity becomes

$$
v_{B}(k)=\sqrt{\frac{2 \pi(1+d) T}{2 d \pi T+d \sqrt{k\left(1-d^{2}\right)+4 \pi^{2} T^{2}}}},
$$

which implies that

$$
\begin{aligned}
v_{B}(k=0) & =\sqrt{\frac{(1+d)}{2 d}} \\
v_{B}(k) & =\sqrt{\frac{(1+d)}{2 d}}+k \frac{(d-1)(d+1)^{\frac{3}{2}}}{32 \pi^{2} \sqrt{2 d} T^{2}}+\mathcal{O}\left(T^{-3}\right),
\end{aligned}
$$

where the first relation was found earlier in [8].

\section{Einstein-Gauss-Bonnet-Maxwell gravity}

Finally, we consider $2+\mathrm{d}$ dimensional planar, spherical, or hyperbolic black hole solutions in Einstein-GaussBonnet-Maxwell gravity. After taking a proper limit, i.e., $k=0$ and/or $q=0$, the result obtained in what follows reproduces all previous results, as they must be reproduced.

The metric is

$$
\begin{aligned}
d s^{2} & =-f(r) N_{\sharp}^{2} d t^{2}+f^{-1} d r^{2}+r^{2} d \Omega_{d}^{2} \\
f(r) & =k+\frac{r^{2}}{2 \lambda}\left[1-\sqrt{1-4 \lambda\left(1+\left(\frac{r}{r_{H}}\right)^{-2 d}\left(q-\frac{\left(\frac{r}{r_{H}}\right)^{-1+d}\left((1+q) r_{H}^{4}+k r_{H}^{2}+k^{2} \lambda\right)}{r_{H}^{4}}\right)\right.}\right] \\
T & =N_{\sharp} \frac{((d+1)+(1-d) q) r_{H}^{4}+(d-1) k r_{H}^{2}+(d-3) k^{2} \lambda}{4 r_{H} \pi\left(r_{H}^{2}+2 k \lambda\right)} .
\end{aligned}
$$

To find the butterfly velocity we solve (5.29) to express the horizon radius $r_{H}$ in terms of the black hole temperature $T$, and then substitute it into (4.24). Since the exact expression is complicated we consider the high-temperature expansion. We find

$$
r_{H}=\frac{4 \pi T}{(1+d)+(1-d) q}+\frac{k[1-d+2((1+q)+d(1-q)) \lambda]}{4 \pi T}+\mathcal{O}\left(T^{-2}\right)
$$

and

$v_{B}\left(\gamma_{G B}\right)=N_{\sharp} \sqrt{\frac{(1+d)+(1-d) q}{2 d}}-N_{\sharp} \frac{k((1+d)+(1-d) q)^{\frac{3}{2}}[1-d+2((1+q)+d(1-q)) \lambda]}{32 \pi^{2} \sqrt{2 d} T^{2}}+\mathcal{O}\left(T^{-3}\right)$.

Let us make the following comments:

(1) From the above results we can see how $\lambda$ and $k$ affect the butterfly velocity.

(2) At a high temperature the butterfly velocities in both spherical and hyperbolic black holes reduce to that in the planar black hole.

(3) The ratio of the butterfly velocities between those with and without the Gauss-Bonnet term is also universal like the planar case mentioned before.

\section{CONCLUSION}

In the first part of this paper we have investigated the butterfly velocity in anisotropic spacetime. We have derived a general formula given in (2.30). We used this formula to study the butterfly velocities in brane systems. We have conjectured that the butterfly velocity is invariant under a double-dimensional reduction generally. In the second part of the paper we studied the butterfly velocity in 
Einstein-Gauss-Bonnet gravity with arbitrary matter fields. The general formula is (4.18). For planar, spherical, or hyperbolic black holes the formula reduces to a simple form (4.22). We have considered several examples by using our formula and discussed several interesting properties.

Let us make the following final comments:

(1) We have conjectured that the butterfly velocity is invariant under a double-dimensional reduction. The implication of such an invariance is not yet understood and it remains to be clarified.

(2) We have found that the ratio of the butterfly velocities between those with and without the Gauss-Bonnet term is universal in the sense that it does not depend on the strength of the Maxwell field. It would be interesting to understand this result better.

(3) It will be interesting to use (4.18) to investigate systems which are not planar, spherical, nor hyperbolic black holes to see how the Gauss-Bonnet term might affect the butterfly velocity.

(4) It will be also interesting find a general butterfly velocity formula in gravity theory with an arbitrary combination of quadratic Riemann curvatures and extend them to anisotropic space [37].

Studies along these lines are in progress.

\section{APPENDIX A: KRUSKAL COORDINATE AND USEFUL RELATIONS}

The line element of time and radial parts for a black hole with a horizon at $r=r_{H}$ is given by

$$
\begin{aligned}
d s^{2} & =-a(r) f(r) d t^{2}+\frac{d r^{2}}{b(r) f(r)} \\
& =-a(r) f(r)\left[d t^{2}-d r_{*}^{2}\right],
\end{aligned}
$$

where $a\left(h_{h}\right) \neq 0, b\left(h_{h}\right) \neq 0$, and $f\left(h_{h}\right)=0$. The tortoise coordinate $r_{*}$ is

$$
d r_{*}=\frac{d r}{f(r) \sqrt{a(r) b(r)}} .
$$

Near the horizon, $f(r) \approx f^{\prime}\left(r_{H}\right)\left(r-r_{H}\right)+\cdots$, we have the relation

$$
\begin{aligned}
r_{*}(r) & =\int_{0}^{r} \frac{d r}{f(r) \sqrt{a(r) b(r)}} \\
& \approx \frac{1}{\sqrt{a\left(r_{H}\right) b\left(r_{H}\right)} f^{\prime}\left(r_{H}\right)} \ln \frac{r-r_{H}}{r_{H}} .
\end{aligned}
$$

Thus, on the horizon the tortoise coordinate $r_{*}\left(r_{H}\right)$ approaches $-\infty$.

The metric can be written in the Kruskal coordinate as follows:

$$
d s^{2}=2 A(u v) d u d v
$$

$$
\begin{aligned}
A(U V) & =\frac{2 a(r) f(r)}{f^{\prime}\left(r_{H}\right)^{2} a\left(r_{H}\right) b\left(r_{H}\right)} e^{-\sqrt{a\left(r_{H}\right) b\left(r_{H}\right)} f^{\prime}\left(r_{H}\right) r_{*}} \\
U & =e^{\sqrt{a\left(r_{H}\right) b\left(r_{H}\right)^{\prime}\left(r_{H}\right)} \frac{f^{\prime}\left(-t+r_{*}\right)}{}} \\
V & =e^{\sqrt{a\left(r_{H}\right) b\left(r_{H}\right)^{\prime}\left(r_{H}\right)} \frac{f^{\prime}\left(t+r_{*}\right)}{}} \\
U V & =e^{\sqrt{a\left(r_{H}\right) b\left(r_{H}\right) f^{\prime}\left(r_{H}\right) r_{*}}} \\
r_{*} & =\frac{1}{\sqrt{a\left(r_{H}\right) b\left(r_{H}\right)} f^{\prime}\left(r_{H}\right)} \ln (U V) .
\end{aligned}
$$

Since the tortoise coordinate $r_{*}\left(r_{H}\right)=-\infty$, we see that $U=0$ on the horizon.

The above definitions and relations imply that

$$
A(U=0)=\frac{2 r_{H}}{f^{\prime}\left(r_{H}\right) b\left(r_{H}\right)},
$$

and

$$
\begin{aligned}
A^{\prime}(U=0) & \left.\equiv \frac{d A(U V)}{d(U V)}\right|_{U=0}=\left.\frac{d A(U V)}{d\left(r_{*}\right)} \frac{d r_{*}}{d(U V)}\right|_{r=r_{H}} \\
& =\left.\frac{2 d\left[a(r) f(r) e^{-\sqrt{a\left(r_{H}\right) b\left(r_{H}\right)} f^{\prime}\left(r_{H}\right) r_{*}}\right]}{f^{\prime}\left(r_{H}\right)^{2} a\left(r_{H}\right) b\left(r_{H}\right) d r_{*}}\left(\frac{1}{\sqrt{a\left(r_{H}\right) b\left(r_{H}\right)} f^{\prime}\left(r_{H}\right)} e^{-\sqrt{a\left(r_{H}\right) b\left(r_{H}\right)} f^{\prime}\left(r_{H}\right) r_{*}}\right)\right|_{r=r_{H}} \\
& =\frac{2 r_{H}^{2}}{b\left(r_{H}\right) f^{\prime}\left(r_{H}\right)}\left(\frac{a^{\prime}\left(r_{H}\right)}{a\left(r_{H}\right)}+\frac{f^{\prime \prime}\left(r_{H}\right)}{f^{\prime}\left(r_{H}\right)}\right),
\end{aligned}
$$

where we have used the relation $f(r) \approx f^{\prime}\left(r_{H}\right)\left(r-r_{H}\right)+\frac{1}{2} f^{\prime \prime}\left(r_{H}\right)\left(r-r_{H}\right)^{2}$. 
Another useful relation is

$$
\begin{aligned}
& \left.h^{\prime}(U=0) \equiv \frac{d h(U V)}{d(U V)}\right|_{U=0}=\left.\frac{d h(U V)}{d(r)} \frac{d r}{d r_{*}} \frac{d r_{*}}{d(U V)}\right|_{r=r_{H}} \\
& =h^{\prime}\left(r_{H}\right)(\sqrt{a(r) b(r)} f(r)) \\
& \times\left(\frac{1}{\sqrt{a_{h} b_{h}} f_{h}^{\prime}} e^{-\sqrt{a_{h} b_{h}} f_{h}^{\prime} r_{*}}\right) \\
& =r_{H} h^{\prime}\left(r_{H}\right) \text {. }
\end{aligned}
$$

\section{APPENDIX B: DERIVATION OF (4.11)}

We first rewrite $\left(R_{U c U d} R^{c d}\right)^{(1)}$ as

$$
\left(R_{U c U d} R^{c d}\right)^{(1)}=R_{U c U d}^{(0)} R^{(1) c d}+R_{U c U d}^{(1)} R^{(0) c d} .
$$

The first term can be easily calculated using

$$
\begin{aligned}
R^{(1) a b} & =\delta^{a V} \delta^{b V}\left(-\left(\frac{2 A^{\prime}}{A^{3}}+\frac{3 d h^{\prime}}{2 A^{2} h}\right) \alpha+\frac{1}{A h} \Delta^{(d)} \alpha\right) \\
R_{U V U V}^{(0)} & =A^{\prime},
\end{aligned}
$$

where Laplacian $\Delta^{(d)} \alpha(x)$ is defined in the d-dimensional metric $d s^{2}=g_{i j} d x^{i} d x^{j}$. We next rewrite the second term as

$$
\begin{aligned}
R_{U c U d}^{(1)} R^{(0) c d} & =R_{U V U V}^{(1)} R^{(0) V V}+R_{U i U j}^{(1)} R^{(0) i j} \\
& =R_{U i U j}^{(1)} R^{(0) i j} .
\end{aligned}
$$

Note that $R_{U V U V}^{(1)}=0$. To calculate the remaining term, $R_{U i U j}^{(1)}$, we write it as

$$
\begin{aligned}
& R_{U i U j}^{(1)}=\left(g_{U a} R^{a}{ }_{i U j}\right)^{(1)} \\
& =g_{U U}^{(1)}\left(R^{U}{ }_{i U j}\right)^{(0)}+g_{\mathrm{UV}}^{(0)}\left(R^{V}{ }_{i U j}\right)^{(1)},
\end{aligned}
$$

and

$g_{U U}^{(1)}\left(R_{i U j}^{(0) U}\right)\left(R^{(0) i j}\right)=\frac{h^{\prime}}{h}\left[R^{(0)}+\frac{2 A^{\prime}}{A^{2}}+\frac{d h^{\prime}}{A h}\right] \alpha(x) \delta(U)$.

The final step is to analyze $\left(R_{i U j}^{V}\right)^{(1)}$. We find

$$
\begin{aligned}
\left(R_{i U j}^{V}\right)^{(1)}= & \nabla_{U} \delta \Gamma_{i j}^{V}-\nabla_{j} \delta \Gamma_{i U}^{V} \\
= & \frac{1}{2} \nabla_{U}\left[g^{V a}\left(\nabla_{i} \delta g_{a j}+\nabla_{j} \delta g_{i a}-\nabla_{a} \delta g_{i j}\right)\right] \\
& -\frac{1}{2} \nabla_{j}\left[g^{V a}\left(\nabla_{i} \delta g_{a U}+\nabla_{U} \delta g_{i a}-\nabla_{a} \delta g_{i U}\right)\right] \\
= & -\frac{1}{2} \nabla_{j} \nabla_{i}\left[g^{V a} \delta g_{a U}\right] .
\end{aligned}
$$

Note that, in the second line, the first three terms are zero, while last two terms are nonzero but they are canceled by each other, and, therefore, only the forth term is left.

Using $\delta g_{a b}=-2 A(U, V) \alpha(x) \delta(U) \delta_{a U} \delta_{b U}$ we obtain a simple relation

$$
\begin{aligned}
\nabla_{j} \nabla_{i}\left(g^{V a} \delta g_{a U}\right)= & -2 R^{(0) i j} \nabla_{i}^{(d)} \nabla_{j}^{(d)} \alpha(x) \delta(U) \\
& +\left[R^{(0)}+\frac{2 A^{\prime}}{A^{2}}+\frac{d h^{\prime}}{A h}\right] \frac{h^{\prime}}{A h} \alpha(x) \delta(U),
\end{aligned}
$$

where the covariant derivative $\nabla_{i}^{(d)}$ is defined in a d-dimensional metric, $d s^{2}=g_{i j} d x^{i} d x^{j}$.

Collecting the above results we find the relation of $\left(R_{U c U d} R^{c d}\right)^{(1)}$ given in (4.10).
[1] A. Almheiri, D. Marolf, J. Polchinski, D. Stanford, and J. Sully, An Apologia for Firewalls, J. High Energy Phys. 09 (2013) 018.

[2] A. Kitaev, Hidden Correlations in the Hawking Radiation and Thermal Noise, in Fundamental Physics Prize Symposium (2014) http://online.kitp.ucsb.edu/online/joint98/.

[3] D. A. Roberts and D. Stanford, Two-Dimensional Conformal Field Theory and the Butterfly Effect, Phys. Rev. Lett. 115, 131603 (2015).

[4] J. Maldacena, S. H. Shenker, and D. Stanford, A bound on chaos, J. High Energy Phys. 08 (2016) 106.

[5] S. H. Shenker and D. Stanford, Black holes and the butterfly effect, J. High Energy Phys. 03 (2014) 067.
[6] S. H. Shenker and D. Stanford, Multiple shocks, J. High Energy Phys. 12 (2014) 046.

[7] S. Leichenauer, Disrupting entanglement of black holes, Phys. Rev. D 90, 046009 (2014).

[8] D. A. Roberts, D. Stanford, and L. Susskind, Localized shocks, J. High Energy Phys. 03 (2015) 051.

[9] S. H. Shenker and D. Stanford, Stringy effects in scrambling, J. High Energy Phys. 05 (2015) 132.

[10] D. A. Roberts and B. Swingle, Lieb-Robinson and the Butterfly Effect, Phys. Rev. Lett. 117, 091602 (2016).

[11] M. Blake, Universal Charge Diffusion and Butterfly Effect, Phys. Rev. Lett. 117, 091601 (2016). 
[12] A. P. Reynolds and S. F. Ross, Butterflies with rotation and charge, Classical Quantum Gravity 33, 215008 (2016).

[13] N. Sircar, J. Sonnenschein, and W. Tangarife, Extending the scope of holographic mutual information and chaotic behavior, J. High Energy Phys. 05 (2016) 091.

[14] W. H. Huang and Y. H. Du, Butterfly effect and holographic mutual information under external field and spatial noncommutativity, J. High Energy Phys. 02 (2017) 032.

[15] X. H. Feng and H. Lu, Butterfly velocity bound and reverse isoperimetric inequality, Phys. Rev. D 95, 066001 (2017).

[16] R. G. Cai, X.X. Zeng, and H. Q. Zhang, Influence of inhomogeneities on holographic mutual information and butterfly effect, J. High Energy Phys. 07 (2017) 082.

[17] V. Jahnke, Delocalizing entanglement of anisotropic black branes, J. High Energy Phys. 01 (2018) 102.

[18] M. Alishahiha, A. Davody, A. Naseh, and S. F. Taghavi, On butterfly effect in higher derivative gravities, J. High Energy Phys. 11 (2016) 032.

[19] E. Caceres, M. Sanchez, and J. Virrueta, Holographic entanglement entropy in time dependent Gauss-Bonnet gravity, J. High Energy Phys. 09 (2017) 127.

[20] M. M. Qaemmaqami, On the butterfly effect in 3D gravity, arXiv:1708.07198.

[21] Y.Z. Li, H. S. Liu, and H. Lu, Quasitopological Ricci polynomial gravities, Phys. Rev. D 96, 106012 (2017).

[22] Y. Ling, P. Liu, and J. P. Wu, Holographic butterfly effect at quantum critical points, J. High Energy Phys. 10 (2017) 025 .

[23] Y. Ling, P. Liu, and J. P. Wu, Note on the butterfly effect in holographic superconductor models, Phys. Lett. B 768, 288 (2017).

[24] D. Giataganas, U. Grsoy, and J. F. Pedraza, Stronglycoupled anisotropic gauge theories and holography, arXiv: 1708.05691 .
[25] M. Blake, R. A. Davison, and S. Sachdev, Thermal diffusivity and chaos in metals without quasiparticles, Phys. Rev. D 96, 106008 (2017).

[26] D. Ahn, Y. Ahn, H. S. Jeong, K. Y. Kim, W. J. Li, and C. Niu, Thermal diffusivity and butterfly velocity in anisotropic Q-Lattice models, J. High Energy Phys. 01 (2018) 140.

[27] K. Sfetsos, On gravitational shock waves in curved spacetimes, Nucl. Phys. B436, 721 (1995).

[28] S. Nojiri and S. D. Odintsov, Brane-world cosmology in higher derivative gravity or warped compactification in the next-to-leading order of AdS/CFT correspondence, J. High Energy Phys. 07 (2000) 049.

[29] T. Dray and G. t'Hooft, Gravitational shock wave of a massless particle, Nucl. Phys. B253, 173 (1985).

[30] T. Ortin, Gravity and Strings, Cambridge Monographs on Mathematical Physics (Cambridge University Press, Cambridge, England, 2004).

[31] D. J. Smith, Intersecting brane solutions in string and M-theory, Classical Quantum Gravity 20, R233 (2003).

[32] D. G. Boulware and S. Deser, String Generated Gravity Models, Phys. Rev. Lett. 55, 2656 (1985).

[33] R. G. Cai, Gauss-Bonnet black holes in AdS spaces, Phys. Rev. D 65, 084014 (2002).

[34] M. Cvetic, S. Nojiri, and S. D. Odintsov, Black hole thermodynamics and negative entropy in de Sitter and anti-de Sitter Einstein-Gauss-Bonnet gravity, Nucl. Phys. B628, 295 (2002).

[35] M. B. Gaete and M. Hassaine, Topological black holes for Einstein-Gauss-Bonnet gravity with a nonminimal scalar field, Phys. Rev. D 88, 104011 (2013).

[36] F. Correa and M. Hassaine, Thermodynamics of Lovelock black holes with a nonminimal scalar field, J. High Energy Phys. 02 (2014) 014.

[37] W. H. Huang (to be published). 